\title{
Comparison of methods for assessing geometric efficiency on multi-detector CT scanners
}

\author{
Theocharis Berris ${ }^{\mathrm{a}, 1}$, Kostas Perisinakis ${ }^{\mathrm{a}, 2}$, Antonios E. Papadakis ${ }^{\mathrm{b}, 3}$, \\ John Damilakis ${ }^{\mathrm{a}, *}$
}

\author{
a University of Crete, Faculty of Medicine, Department of Medical Physics, P.O. Box 2208, 71003 Heraklion, Crete, Greece \\ b University Hospital of Heraklion, Department of Medical Physics, P.O. Box 1352, 71110 Heraklion, Crete, Greece
}

Received 21 October 2011; received in revised form 11 April 2012; accepted 14 April 2012

Available online 5 May 2012

\section{KEYWORDS \\ Radiation dosimetry; \\ MDCT; \\ Geometric efficiency; \\ Quality control}

\begin{abstract}
The aim of the current study was to compare the film method against the method based on a new CT slice detector in assessing geometric efficiency (GE) of $x$-ray beams utilized by a multi-detector CT (MDCT) scanner. Measurements of GE were performed using radiographic films and a solid state CT slice detector for all beam qualities, collimations and focal spot sizes available on an MDCT scanner. Repeatability of GE measurements was assessed. The radiographic film and the solid state detector methods were compared to each other in regard to efficacy in measuring free-in-air GE. The values of GE determined using the radiographic film method were found to range between $48.5 \%$ and $90.6 \%$. Differences between values obtained with the radiographic film method and corresponding values obtained with the solid state detector were less than $10 \%$ exceeding $5 \%$ for only one case. Both methods show that wide beams have higher GE values compared to thin ones. The use of large instead of small focal spot was found to deteriorate GE values by up to $23.1 \%$. Beam quality did not seem to influence GE of the various collimations. When thin beam collimations are employed, a considerable amount of the radiation is wasted for non-imaging purposes. Both film and solid state probe methods are capable of measuring GE of thin as well as wide collimations. The solid state detector is the easiest to use, however its usefulness is reduced by the fact that it cannot measure dose profiles of beam collimations available for step-and-shoot mode of operation. (C) 2012 Associazione Italiana di Fisica Medica. Published by Elsevier Ltd. All rights reserved.
\end{abstract}

\footnotetext{
* Corresponding author. Tel.: +30 2810 392569; fax: +30 2810394933.

E-mail addresses: theocharisberris@yahoo.com (T. Berris), perisina@med.uoc.gr (K. Perisinakis), apapadak@edu.med.uoc.gr (A.E. Papadakis), damilaki@med.uoc.gr (J. Damilakis).

${ }^{1}$ Present address: International Atomic Energy Agency, Department of Nuclear Safety and Security, NSRW, RSM, Radiation Protection of Patients Unit, Vienna International Centre, PO Box 100, A-1400 Vienna, Austria. Tel.: +4312600 26474.

2 Tel.: +302810392564 .

${ }^{3}$ Tel.: +302810392092 .
}

1120-1797/\$ - see front matter ( $\odot 2012$ Associazione Italiana di Fisica Medica. Published by Elsevier Ltd. All rights reserved. http://dx.doi.org/10.1016/j.ejmp.2012.04.003 


\section{Introduction}

In multi-detector CT (MDCT) scanners, calibration problems may arise when the outmost elements of the detector array are exposed to beam penumbra [1]. Differences in x-ray intensity along the detector rows may lead to differences in section sensitivity profiles and cause image artifacts. Manufacturers increase the $x$-ray beam width along the $z$-axis so that penumbra falls outside the active detector array. As a consequence, a fraction of the $x$-ray beam does not contribute to image formation, just increasing patient radiation dose burden. This effect has been described in literature as overbeaming [2,3]. A simple means to quantify overbeaming in MDCT scanners is free-in-air geometric efficiency (GE). The International Electrotechnical Commission (IEC) has defined GE, as the integral of the dose profile over the nominal active detector array width, divided by the total integral of the dose profile $[4,5]$. The ever growing utilization of CT in clinical practise [6-8] renders investigation of all dosimetric aspects of CT scanners imperative. GE comprises an important exposure feature of CT scanners. A recent study showed that decreased GE is correlated with increased patient radiation dose [9].

Two methods have been commonly employed to determine CT dose profiles along $z$-axis i.e. radiographic films [10] and thermoluminescence dosimeter (TLD) arrays [11]. Radiation profiles have also been obtained using computed radiography (CR) systems [12] and a recently developed solid state CT slice detector [9]. The aim of this study was to compare the method based on radiographic film for assessing GE of the beams utilized by an MDCT scanner against the corresponding method based on a new solid state detector dedicated for CT dosimetry and demonstrate their advantages and disadvantages. The present study is complementary to the previous work presented elsewhere [9]. The previous study was focused in shedding light into the exposure parameters that affect GE and the effect of GE on patient dose, whereas the present study is focused on technical issues regarding the measurement of GE with different methods to reveal advantages and disadvantages of each method.

\section{Materials and methods}

\section{CT scanner}

A Siemens SOMATOM Sensation 16-slice CT scanner (Siemens AG, Forchheim, Germany) was used in the present study. This scanner can be operated in body and head mode at 80 , 120 and $140 \mathrm{kVp}$ nominal tube voltage values. Available beam collimations and half value layers (HVL) of CT beams are shown in Tables 1 and 2 respectively. Focal spot size automatically swaps from $0.5 \times 0.7 \mathrm{~mm}^{2}$ to $0.8 \times 1.2 \mathrm{~mm}^{2}$ when tube current exceeds a threshold which depends on the mode of operation [13].

\section{Determination of free-in-air geometric efficiency using radiographic films}

To correctly measure the dose profiles of $\mathrm{CT}$ beams with radiographic film, the film must be calibrated in the different
Table 1 Available beam collimations for head and body mode at $80, \quad 120$ and $140 \mathrm{kVp}$ (number of slices $\times$ reconstruction width in $\mathrm{mm}$ ), for the Siemens Sensation 16-slice CT scanner.

\begin{tabular}{lc}
\hline Small focus & Large focus \\
\hline $16 \times 1.5=24$ & $16 \times 1.5=24$ \\
$12 \times 1.5=18$ & $12 \times 1.5=18$ \\
$16 \times 0.75=12$ & $16 \times 0.75=12$ \\
$2 \times 5=10$ & $2 \times 5=10$ \\
$12 \times 0.75=9$ & $12 \times 0.75=9$ \\
$2 \times 1=2$ & $2 \times 1=2$ \\
$2 \times 0.6=1.2^{\mathrm{a}}$ & N/A \\
\hline
\end{tabular}

N/A: Not available.

a Not available in head mode, $140 \mathrm{kV}$.

beam qualities produced by the scanner. However, due to the incapability of the CT scanner to yield low doses needed for low dose range film calibration, the CT beam qualities were reproduced on an $\mathrm{x}$-ray unit capable of producing all the different dose levels (low, high and intermediate) required for calibration. A square area was irradiated on every film used. The optical density (OD) at the centre of the irradiated area of the films was then plotted against dose on the films which was measured using a multi-purpose detector at the same point. As a next step, an optical scanner was used to digitize the irradiated films. The pixel values of irradiated areas (of known OD) were measured with image processing software and plotted against OD. Curves were fitted to the data and the film and optical scanner calibration equations were produced. In order to be able to measure dose profiles from digitized films irradiated on CT, OD was algebraically eliminated between the scanner calibration equation and each of the film calibration equations for different beam qualities. The resulting equations were functions of dose against pixel value. This was achieved by the use of appropriate mathematical software. Finally, the irradiated films on the CT scanner containing profiles of different collimations and beam qualities were digitized and the pixel value profile of each beam was obtained by using the image processing software. By using the appropriate 'pixel value versus dose' equation these pixel values profiles were transformed to dose profiles for that particular collimation.

\section{Film calibration}

All CT scanner beam qualities were reproduced on a Siemens SIREGRAPH C (Siemens AG, Forchheim, Germany) radiographic unit by adding aluminium plates to the beam exit window. The HVL of the beams was verified with the multi-purpose detector of a calibrated Barracuda x-ray multimeter (RTI Electronics, AB, Mölndal, Sweden).

Table 2 Beam qualities of available beams in the Siemens Sensation 16 CT scanner.

\begin{tabular}{lll}
\hline Tube voltage $(\mathrm{kVp})$ & \multicolumn{2}{l}{ Half value layer $(\mathrm{mm} \mathrm{Al})$} \\
\cline { 2 - 3 } & Body mode & Head mode \\
\hline 80 & 6.3 & 5.0 \\
120 & 8.8 & 7.5 \\
140 & 9.6 & 8.7 \\
\hline
\end{tabular}


Radiographic films (Fuji HR U-30, $24 \times 30 \mathrm{~cm}^{2}$, FUJIFILM Medical Systems USA, Inc., Stamford, CT) contained in a light-tight envelope, were exposed to beams of incrementally increasing tube load. A $10 \times 10 \mathrm{~cm}^{2}$ field and $100 \mathrm{~cm}$ source-to-surface distance was selected for all exposures. The corresponding free-in-air doses were measured by exposing the multi-purpose detector at the same settings. An optical densitometer (pehamed DENSOQUICK 2, PEHA med. GeräteGmbH, Sulzbach, Germany) was used to measure optical density of the uniformly irradiated area of the developed films. OD versus free-in-air dose curves were derived for all available beam qualities.

\section{Collection of dose profiles along z-axis}

Radiographic films in a light-tight paper envelope were used to obtain CT dose profiles at the isocentre, for all available beam qualities and collimations of the 16-slice CT scanner studied. Films were positioned with their longest dimension along the z-axis. The centre of every film coincided with the CT scanner isocentre. To avoid backscattering originating from the scanner bed, the light-proof envelope was placed on a cardboard extension of the bed. All measurements were performed with the x-ray tube stationary at "12 o'clock". The tube load was limited to the minimum, namely 49, 33 and $27 \mathrm{mAs}$ for 80,120 and $140 \mathrm{kVp}$ respectively.

\section{Optical scanner calibration, film digitization and} derivation of dose versus grey level equations

Film digitization was performed using a Microtek ScanMaker 9600XL (Science-based Industry Park, Hsinchu, Taiwan) scanner, capable of handling transparent media. Films exposed to incrementally increasing tube voltage and load were used to determine the relationship between OD and corresponding grey level values. Greyscale 8-bit images of the dose profiles were obtained. The spatial resolution of the generated images was set to $300 \mathrm{dpi}$ in order to be comparable to the resolution of dedicated film digitizers [14] while maintaining a logical image file size.

Mathematica ${ }^{\circledR} 7$ (Wolfram research, Champaign, IL, USA) was used for the required symbolic mathematical manipulations. Mathematical expressions of free-in-air dose versus grey levels were derived for every beam quality by eliminating OD between film calibration and optical scanner calibration fitted equations.

\section{Determination of free-in-air geometric efficiency}

Image-Pro Plus 4.1 (Media Cybernetics Inc., Bethesda, MD, USA) was used to acquire the profile pixel values (greylevels) from the digitized film images. Pixel values were imported to OriginPro ${ }^{\circledR} 7.0$ (OriginLab Corporation, Northampton, MA, USA) spreadsheets. Applying the dose versus grey-level equations to the pixel values, dose profiles along $z$-axis were obtained. GE was calculated by the expression:

$$
G E_{\text {free-in-air }}=\frac{\int_{-a / 2}^{+a / 2} D(z) \mathrm{d} z}{\int_{-\infty}^{+\infty} D(z) \mathrm{d} z} \cong \frac{\int_{-a / 2}^{+a / 2} D(z) \mathrm{d} z}{\int_{-140 \mathrm{~mm}}^{+140 \mathrm{~mm}} D(z) \mathrm{d} z}
$$

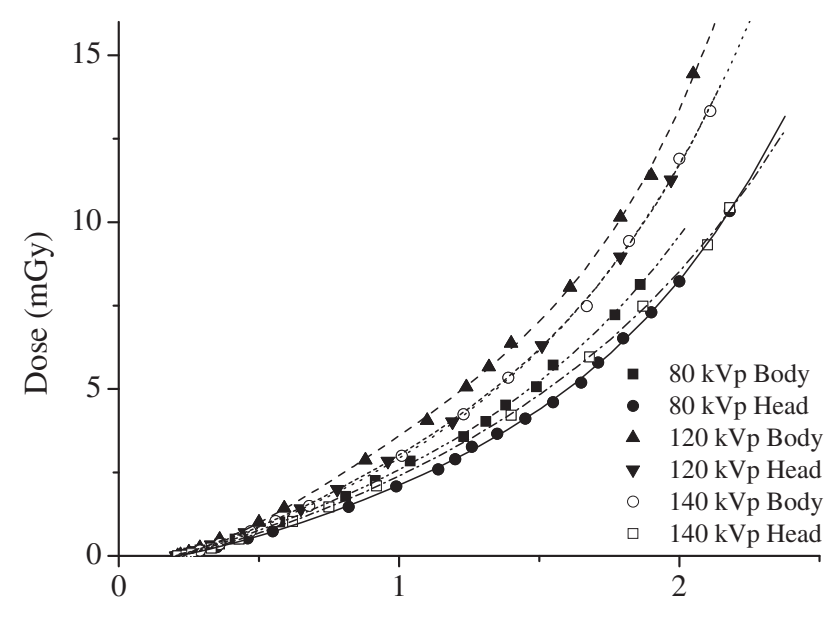

OD

Figure 1 Fourth order polynomial curves fitted to the film calibration data. The figure depicts curves for all available beam qualities.

where $D(z)$ is the dose value along $z$-axis, $a$ is the nominal beam width along $z$-axis at the isocentre, $\int_{-a / 2}^{+a / 2} D(z) d z$ is the dose integral corresponding to the nominal detector width and $\int_{-\infty}^{+\infty} D(z) \mathrm{d} z \cong \int_{-140 \mathrm{~mm}}^{+140 \mathrm{~mm}} D(z) \mathrm{d} z$ is the total dose integral. The integration interval ranged from -140 to $140 \mathrm{~mm}$, despite the fact that the film was $30 \mathrm{~cm}$ long, in order to create a margin for film centering errors.

\section{Determination of free-in-air geometric efficiency using a CT slice detector}

The $x$-ray multimeter coupled with the CT-SD16 slice detector (RTI Electronics, AB, Mölndal, Sweden) was employed to determine the dose profiles for several available beam collimations. CT-SD16 is especially designed for CT dose measurements. It consists of an aluminium tube

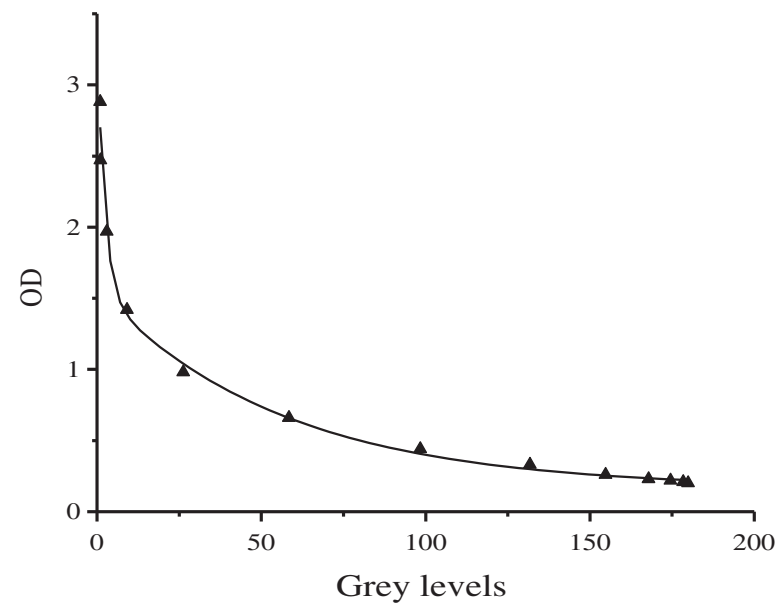

Figure 2 The calibration curve determined for the optical scanner used in this study. 

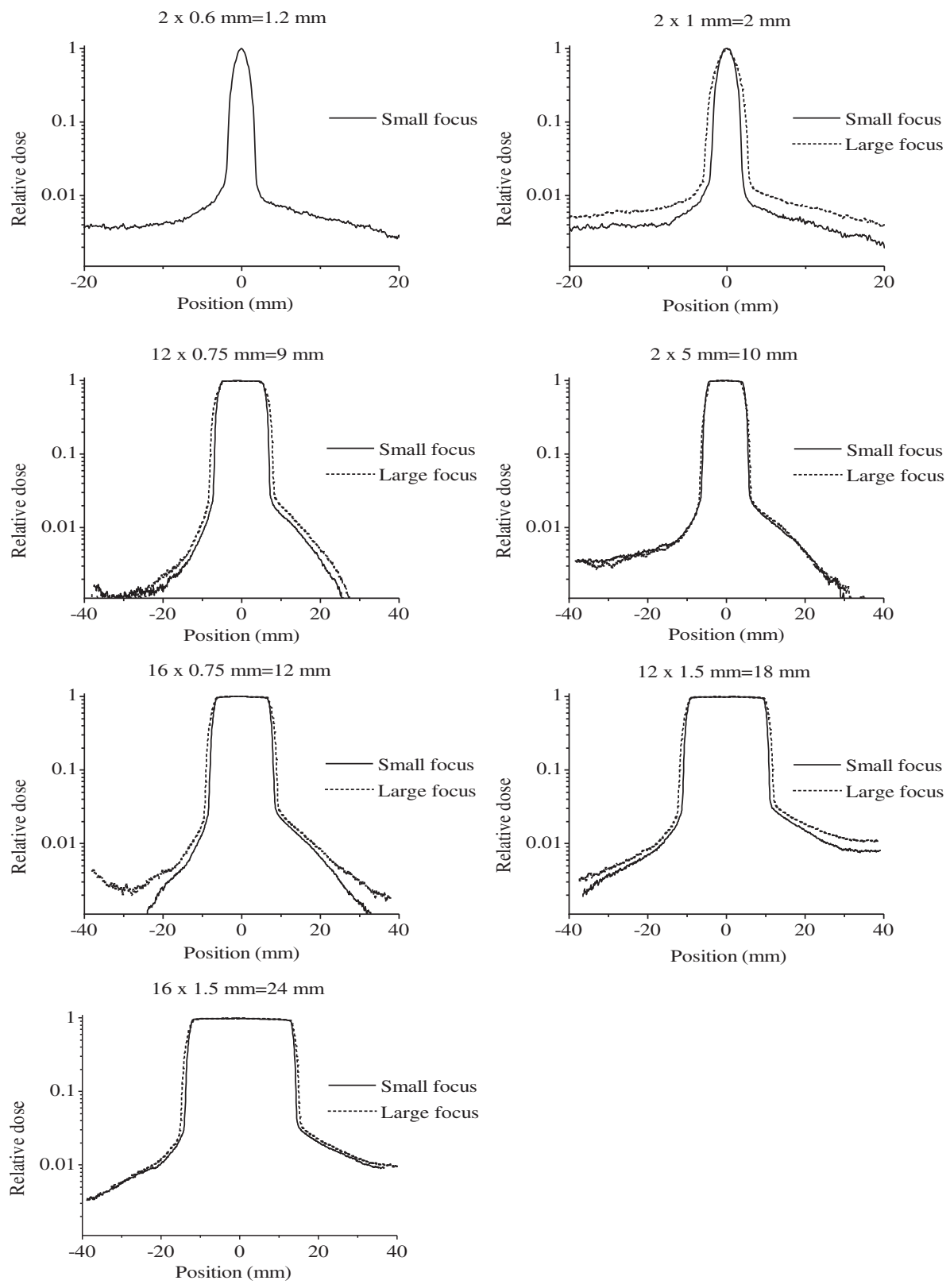

Figure 3 Free-in-air dose profiles measured with the film method at $80 \mathrm{kVp}$, body mode for the available collimations. Profiles produced by the small and the large focal spot are shown.

housing which encloses a very thin ( $250 \mu \mathrm{m}$ along $z$-axis) solid state detector at its centre. The solid state detector tube was supported by a custom-made cardboard extension beyond the edge of the scanner table in order to avoid interference of the table to detector exposure and positioned at the isocentre coaxial with the z-axis of the CT scanner. As the table moved along z-axis during a spiral acquisition, the intensity of the $x$-ray beam incident to the detector was monitored. The detector has a sampling rate of 2000 dose values per second. Free-in-air dose profiles at the isocentre were obtained for all collimations available in spiral mode, namely $16 \times 1.5 \mathrm{~mm}=24 \mathrm{~mm}$, $16 \times 0.75 \mathrm{~mm}=12 \mathrm{~mm}$ and $2 \times 0.6 \mathrm{~mm}=1.2 \mathrm{~mm}(1.2 \mathrm{~mm}$ is available for the small focal spot size only). As a result of the CT-SD16 probe design and function, it was not possible to measure dose profiles for beam collimations selectable only in sequential mode, which require the scanner table to remain stationary during the exposure.

The software accompanying the CT-SD16 detector provides the user with information about the beam's full width at half maximum (FWHM), $\mathrm{CTDI}_{100}$ and $\mathrm{CTDI}_{\text {vol }}$. The capability of exporting dose profiles to a spreadsheet is also provided. Free-in-air GE for all available beam qualities and collimations was calculated from the dose profiles, using Equation (1).

\section{Repeatability of free-in-air geometric efficiency measurement methods}

The repeatability of a) film and b) solid state detector measurement methods was assessed in terms of standard 

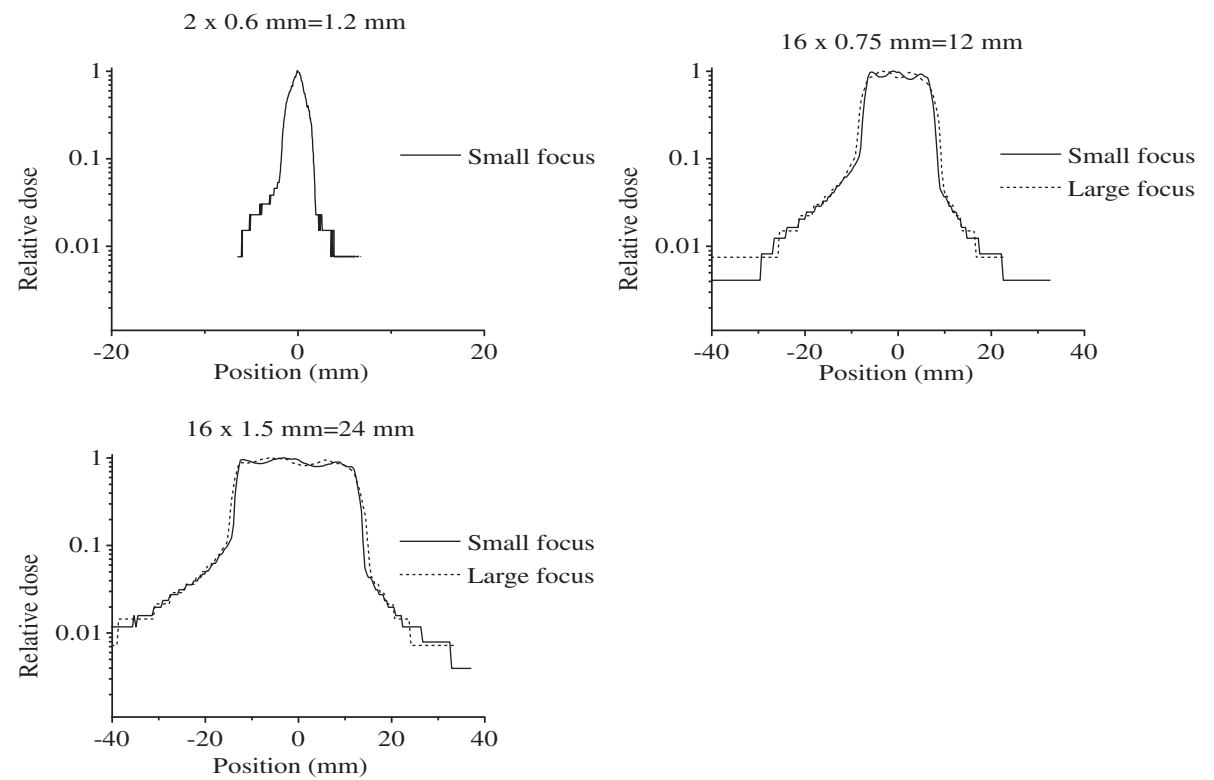

Figure 4 Free-in-air dose profiles measured with the CT-SD16 detector method at $80 \mathrm{kVp}$, body mode for the available collimations. Profiles produced by the small and the large focal spot are shown.

deviation by repeated measurements of GE for all available collimations at $80 \mathrm{kVp}$ body mode. Five measurements were performed for every collimation. The whole procedure was repeated for every method, including irradiations and analysis of collected dose data.

\section{Results}

Film calibration curves for all beam qualities are depicted in Fig. 1. Fourth order polynomial equations fitted the data well. The $R^{2}$ coefficient value was greater than 0.999 for all equations. The optical scanner calibration curve is shown in Fig. 2. In this case the $R^{2}$ coefficient for the exponential equation fitted to the data was 0.993 .

Free-in-air dose profiles were obtained for all beam qualities and collimations using a) radiographic films and b) the CT-SD16 detector. The range of peak ODs for the films irradiated on the CT scanner was 1.23-1.99. Figures 3 and 4 show profiles collected for $80 \mathrm{kVp}$ body mode of operation using films and the solid state detector respectively. Profiles obtained for large and small focal spot size are superimposed for comparison. The profiles are plotted in semi-logarithmic scale in order to exemplify the low dose "tails" of the profiles. As it is evident in Figs. 3 and 4, the dose profile values were not symmetrical in regard to their central value. Spatial sampling for film measurements was $300 \mathrm{dpi}$ or 11.8 points $/ \mathrm{mm}$. The spatial sampling of the solid state detector was limited by the finite thickness of the detector (i.e. $0.25 \mathrm{~mm}$ ).

Tables 3 and 4 illustrate GE values for all available collimations, beam qualities and focal spot sizes for body and head mode respectively. Measurements with film and the CT-SD16 probe are shown alongside for direct

Table 3 Geometric efficiencies (\%) for all available beam qualities and collimations in body mode, measured with the film method and the CT-SD16 solid state detector method.

\begin{tabular}{|c|c|c|c|c|c|c|c|}
\hline \multirow[t]{2}{*}{ Collimation (mm) } & \multirow[t]{2}{*}{ Measurement method } & \multicolumn{2}{|c|}{$80 \mathrm{kVp}$} & \multicolumn{2}{|c|}{$120 \mathrm{kVp}$} & \multicolumn{2}{|c|}{$140 \mathrm{kVp}$} \\
\hline & & SF & LF & SF & LF & SF & LF \\
\hline \multirow[t]{2}{*}{1.2} & Film & 50.9 & N/A & 52.2 & N/A & 48.5 & N/A \\
\hline & CT-SD16 & 48.5 & N/A & 47.8 & N/A & N/A & $\mathrm{N} / \mathrm{A}$ \\
\hline 2 & Film & 70.0 & 55.9 & 70.2 & 57.3 & 69.9 & 55.1 \\
\hline 9 & Film & 74.4 & 69.4 & 73.0 & 67.6 & 74.1 & 68.1 \\
\hline 10 & Film & 89.2 & 86.8 & 90.6 & 87.1 & 89.2 & 87.3 \\
\hline \multirow[t]{2}{*}{12} & Film & 77.5 & 72.6 & 77.4 & 72.7 & 77.0 & 72.1 \\
\hline & CT-SD16 & 75.7 & 71.9 & 75.6 & 70.0 & 75.8 & 70.2 \\
\hline 18 & Film & 83.2 & 81.0 & 84.8 & 81.3 & 84.1 & 79.9 \\
\hline \multirow[t]{2}{*}{24} & Film & 86.2 & 83.3 & 85.7 & 82.6 & 86.2 & 82.3 \\
\hline & CT-SD16 & 86.2 & 83.4 & 84.9 & 81.5 & 84.0 & 82.4 \\
\hline
\end{tabular}

SF: Small focus; LF: Large focus; N/A: Not available. 
Table 4 Geometric efficiencies (\%) for all available beam qualities and collimations in head mode, measured with the film method and the CT-SD16 solid state detector method.

\begin{tabular}{|c|c|c|c|c|c|c|c|}
\hline \multirow[t]{2}{*}{ Collimation (mm) } & \multirow[t]{2}{*}{ Measurement method } & \multicolumn{2}{|c|}{$80 \mathrm{kVp}$} & \multicolumn{2}{|c|}{$120 \mathrm{kVp}$} & \multicolumn{2}{|c|}{$140 \mathrm{kVp}$} \\
\hline & & SF & LF & SF & LF & SF & LF \\
\hline \multirow[t]{2}{*}{1.2} & Film & 50.6 & N/A & 49.2 & N/A & N/A & N/A \\
\hline & CT-SD16 & 48.3 & N/A & 48.8 & N/A & $\mathrm{N} / \mathrm{A}$ & $\mathrm{N} / \mathrm{A}$ \\
\hline 2 & Film & 68.2 & 54.3 & 69.2 & 53.2 & 67.3 & 56.0 \\
\hline 9 & Film & 75.5 & 66.9 & 74.9 & 68.8 & 74.1 & 69.1 \\
\hline 10 & Film & 89.3 & 85.8 & 90.2 & 87.4 & 89.2 & 87.1 \\
\hline \multirow[t]{2}{*}{12} & Film & 77.5 & 73.0 & 78.5 & 72.3 & 77.8 & 71.9 \\
\hline & CT-SD16 & 74.6 & 71.0 & 75.3 & 69.9 & $\mathrm{~N} / \mathrm{A}$ & N/A \\
\hline 18 & Film & 84.0 & 79.5 & 86.8 & 82.1 & 85.0 & 81.0 \\
\hline \multirow[t]{2}{*}{24} & Film & 87.7 & 84.0 & 88.7 & 83.6 & 86.0 & 83.1 \\
\hline & CT-SD16 & 84.7 & 83.5 & 85.7 & 82.5 & $\mathrm{~N} / \mathrm{A}$ & N/A \\
\hline
\end{tabular}

SF: Small focus; LF: Large focus; N/A: Not available.

comparison. The values of GE measured with film, ranged from $48.5 \%$ to $90.6 \%$ for body mode and similarly from $48.3 \%$ to $90.2 \%$ for head mode of operation. As shown in Fig. 5 the differences of the values of GE determined with film from the corresponding values determined with the CT-SD16 detector did not exceed 5\%, except for one of all the available collimations. Figure 6 shows superimposed dose profiles in semi-logarithmic scale obtained with both measurement techniques for the selectable collimations at $80 \mathrm{kVp}$, body mode.

Wider beams were found to have higher values of GE than the thinner ones. The only exception was the $10 \mathrm{~mm}$ wide collimation which had the highest GE value disregarding the general pattern. Increasing focal spot size was found to be associated with a deterioration of GE, which was more pronounced for thinner beam collimations. Figure 7 illustrates GE values for the available collimations at $80 \mathrm{kVp}$ body mode acquired with film and the solid state probe. As shown in the insets, when the large instead of the small focal spot was employed, a decrease of up to $20.1 \%$ was recorded. Geometric efficiency was found to be insensitive to beam quality by both methods of measurement. Figure 8 illustrates the GE values for every beam quality and the small focal spot size in use. Values obtained with the large focal spot were also insensitive to beam quality.

Repeatability of film measurements was found not to exceed $2.2 \%$ for any of the collimations tested. The repeatability of CT-SD16 measurements was estimated to be up to $1.1 \%$, which was calculated for the $12 \mathrm{~mm}$ collimation and large focal spot.

\section{Discussion}

The efficiency of radiation utilization in MDCT systems is expressed in terms of free-in-air GE. GE has been recognized as an important parameter and for this reason it has been proposed to manufacturers to provide the users with an estimate of GE each time the parameter assumes values lower than 70\% [15]. Few studies concerning the measurement of GE in MDCT scanners exist in the literature. Besides, there is no study to our knowledge, on comparing available methods for the determination of GE. In the
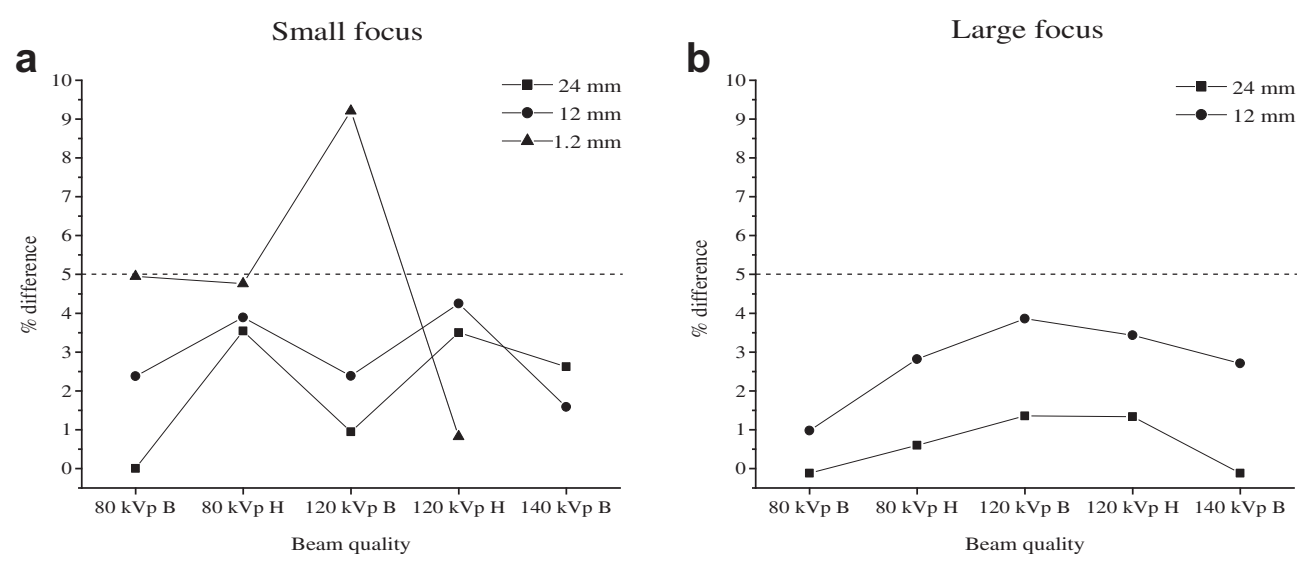

Figure 5 Percentage differences between corresponding values of GE determined with the film method and the CT-SD16 detector method for the a) Small focal spot, and b) Large focal spot. Dashed lines mark the 5\% difference level. Measurements were performed for all available tube voltages in body $(\mathrm{B})$ and head $(\mathrm{H})$ mode of operation. 

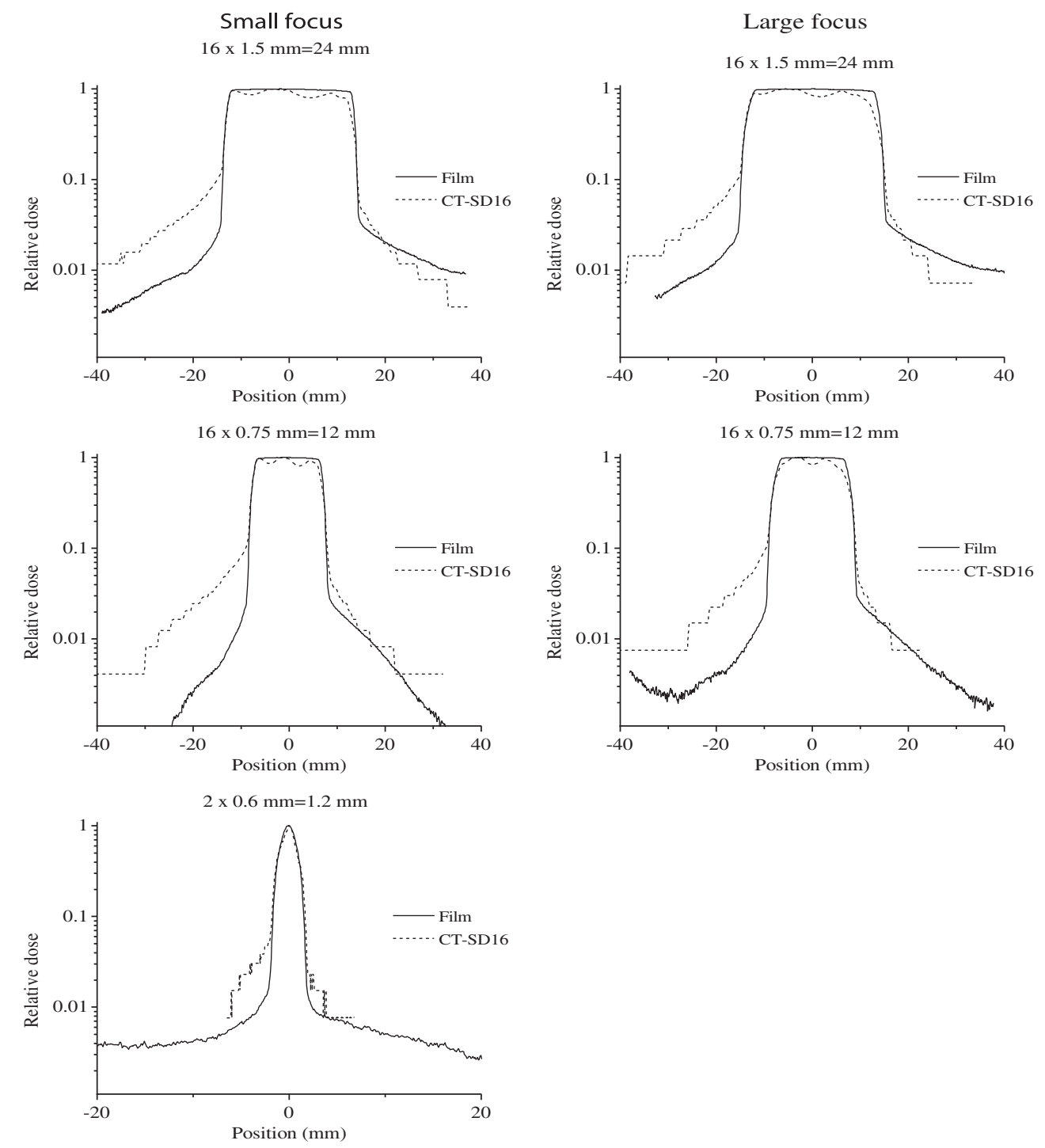

Figure 6 Profiles obtained with the film method and the solid state detector method for all available collimations at $80 \mathrm{kVp}$, body mode, spiral acquisition. The left and right columns of figures depict profiles obtained with the small and large focal spot in use respectively.

current study GE values for beam collimations available on an MDCT scanner were determined using a) film and b) a solid state probe. These methods for the determination of GE were compared to demonstrate advantages and disadvantages. The authors acknowledge the limitation arising from the use of a relatively old CT system in the experimental process. Nevertheless, 16 slice CT scanners are all purpose and their principle of operation is similar to that of MDCT systems with more than 16 detector rows. Measurements of GE would also follow the same principles disregarding the number of detector rows. This is expected to be valid even for scanners with $\geq 64$ detector rows. An approach which would help in reducing overbeaming in MDCT scanners was proposed in the early 2000s. A real time tracking system would move the collimators and align the detectors and the beam (refreshing every $20 \mathrm{~ms}$ ). For the particular scanner tested the reduction of dose was up to $40 \%$. The method was found to have the potential to provide dose efficiency comparable with that of single detector row CT scanners [16]. However, the literature is very poor regarding the issue of correcting for reduced GE although it looks like even scanners at the highest end of CT technology suffer from reduced utilization of radiation on the $z$-axis due to reduced GE [17]. It should be mentioned that reported GE values refer to maximum theoretical values, given that the measured dose profile were positioned centrally on the detector array i.e. ideally. Miscentering would cause reduction of GE. Besides, x-ray utilization efficiency of a CT scanner cannot be estimated taking into account only the measured GE values. The dead space between detector elements and the $\mathrm{x}$-ray absorption efficiency of the detector are also factors affecting x-ray utilization fraction. The total dead space between detector elements is expected to increase with increasing number of detector rows, while x-ray absorption efficiency depends on the material and the size of each detector element. Apart from geometric efficiency, both total dead space and $\mathrm{x}$-ray absorption efficiency should be determined and taken 

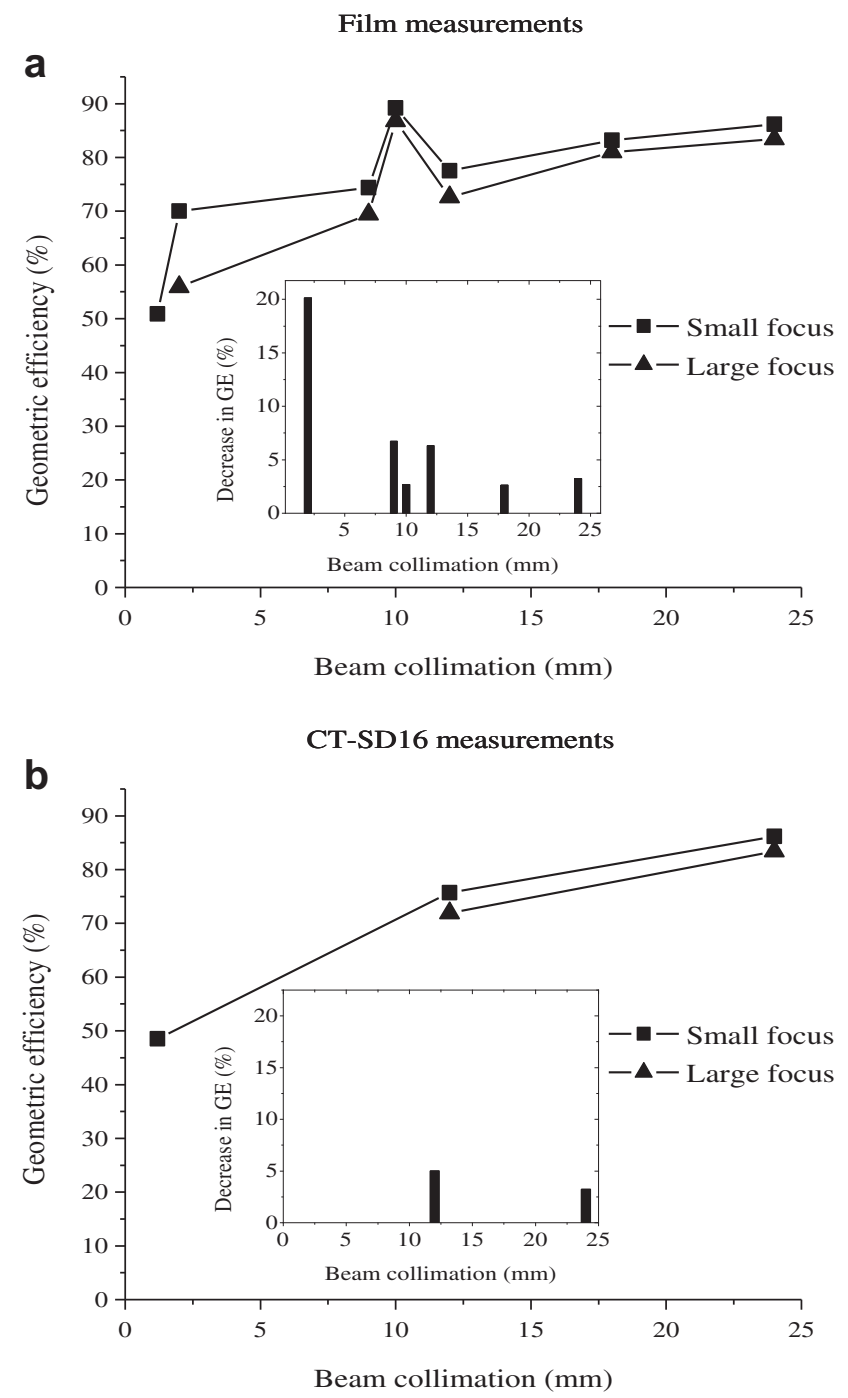

Figure 7 GE for $80 \mathrm{kVp}$, body mode of operation measured by a) Film and b) The CT-SD16 detector. Insets exemplify the percent variance in GE caused by focal spot swap.

into account if a reliable x-ray utilization factor is to be derived.

A collimated CT beam with a GE of $50 \%$ means that half of the patient radiation burden is wasted for non diagnostic purposes. This study has shown that thin collimations may be associated with a GE of less than $50 \%$. GE was found to depend on the geometrical characteristics of the $x$-ray beam. As expected wider beams tend to have higher GE values than the thin ones. This may be attributed to the fact that for thinner collimations, the penumbral region is a relatively larger portion of the total beam width than for wider beams. The exception observed for the $10 \mathrm{~mm}$ beam has been explained in previous studies $[9,10]$. Focal spot size was also found to affect GE values. As shown in Figs. 3 and 4 beams produced using the large focal spot had wider penumbras than those originating from the small focal spot and therefore had lower GE values. Beam quality which depends on the mode of operation and tube voltage had no observable effect on GE. The asymmetry observed in the nominal beam width of the profiles (high dose area) might be due to the heel effect which has been observed for beams as wide as $10 \mathrm{~mm}$ [18]. The right "tails" of the profiles acquired with film were found to be more elevated than the corresponding left "tails". This may be due to some structural asymmetry of the collimators. The profiles obtained with the solid state detector could also be affected by the probably present heel effect as film measurements indicated. In the case of the solid state detector the high dose part of the profiles demonstrated a tendency to follow the heel effect which was observed for film. However, in the case of the solid state detector the results may have been also affected by the imperfectly corrected existing angular dependence of the solid state detector. The manufacturer claims that the CT-SD16 probe demonstrates angular dependence for beams wider than $5 \mathrm{~mm}$. If the probe is scanned towards the connector side a mathematical correction may be applied from the acquisition software right after acquisition. This is exactly what was followed in this study. Another factor which may have affected the solid state profiles might be imperfect matching of the axis of the solid state detector to the $z$-axis of the CT scanner while positioning the detector for measurements.

Radiographic film is frequently used in radiology departments. Due to its relatively low cost, film dosimetry is commonly employed in radiation measurements. Assessment of GE with radiographic film is effectively straightforward and comprehensible. The current study has shown that a common optical scanner in conjunction with customary software can be used for the determination of $\mathrm{GE}$ in MDCT. Also, the spatial sampling of film measurements is adequate to provide detailed dose profiles such as those provided by the relatively expensive [14] dedicated film scanners. Nevertheless film measurements are tedious and time consuming. They require preparation consisting of a series of calibrations in order to perform the measurement. Elaborate processing after measurement is also required to obtain the results. This method requires caution during film exposures. OD should be kept below 2 in order to be well within the linear region of the sensitometric curve of the film used. Optical scanner saturation constitutes an equally important issue. The scanner is incapable of distinguishing between ODs exceeding a threshold value. This could result in dose profile clipping, i.e. doses corresponding to the saturated region of the digitized image will appear to be lower than they really are. The saturation threshold of the optical scanner used in the present study was assessed to be around a value of OD higher than 2 . Another drawback of the film method is that film cannot be reused.

The CT-SD16 solid state detector is a specialized piece of equipment used in CT dosimetry. The measurement software is easy to use and dose profiles are produced automatically without preparation. Spatial resolution of the obtained profiles is also high for the CT-SD16 probe. GE values are easily calculated from the obtained profiles using scientific software. A drawback of the CT-SD16 is its inability to measure dose profiles of beams available only in sequential mode.

Measurements of free-in-air GE of the same CT scanner used in the current study have been previously performed with TLD arrays [9]. The arrays consisted of stacks of TLD 

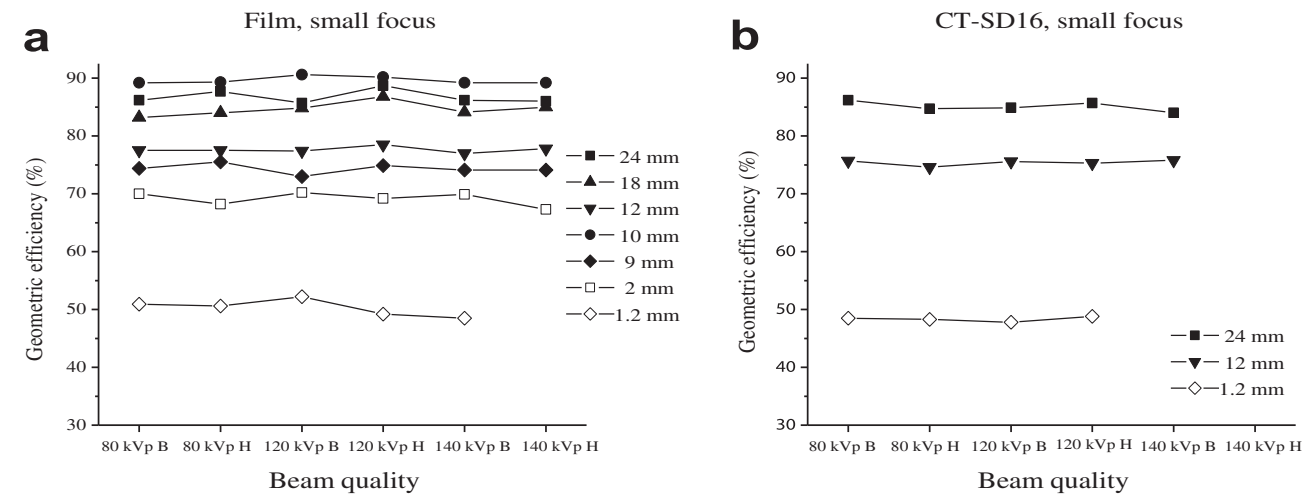

Figure 8 GEs for every available collimation and nominal tube voltage for body (B) and head $(\mathrm{H})$ mode of operation. Measurements were performed with a) Radiographic film and b) The solid state detector. Tube load and voltage were adjusted appropriately so that only the small focal spot was in use.

chips $0.9 \mathrm{~mm}$ thick, positioned back to back. Table 5 illustrates a comparison between the GE values obtained with film measurements performed in this study and the corresponding TLD measurements published elsewhere [9]. The values under comparison were obtained at 80 and $120 \mathrm{kVp}$ body mode, for large and small focus. Figure 9 shows the percentage difference of values measured with the TLD method from the corresponding values measured with radiographic film. Both methods yielded fairly comparable results for wider collimations. Larger discrepancies observed at thinner collimations (i.e. 2 and $1.2 \mathrm{~mm}$ ) could be attributed to the decreased spatial sampling of the TLD arrays. Measuring GE with TLDs is a time consuming process. This method needs more time than films and much more time and effort than measurements with the solid state detector. Data reported by ImpaCT organization was also presented in Table 5 for comparison purposes. Differences between GE values estimated using film and corresponding values reported by ImpaCT group were up to $7 \%$ for beams produced using small focal spot and up to $10 \%$ for beams produced using the large focal spot. It is noted that ImpaCT-reported GE values have been derived for the same scanner model but a different scanner.
During the experimental process of the current study, measurement of free-in-air GE using CR equipment was attempted. An Agfa CR 75.0 ${ }^{\mathrm{TM}}$ digitizer (Agfa Medical Systems, Ridgefield Park, NJ) in conjunction with Agfa MD$40 \mathrm{CR}$ cassettes was employed. CR screens were placed in a light-tight paper envelope to reduce widening of the beam due to scattering arising from the plastic cassette. Exposures were performed using the lowest tube loads available. The experimental setup was identical with that of film measurements. The CT scanner under investigation was incapable of producing tube loads below $27 \mathrm{mAs}$. As a result, the exposed CR screens were always saturated and the radiation profiles were clipped. Nevertheless, as demonstrated elsewhere [12], measurement of CT radiation profiles using CR equipment is feasible. As long as saturation of the detector is avoided, radiation profiles can be obtained. The detail of the acquired profiles should be comparable to that of dose profiles obtained with film or the solid state detector as spatial sampling of CR plates may reach 20 points $/ \mathrm{mm}$ for high resolution $C R$ screens. Provided the equation of pixel value versus exposure is also known for the CR system in question and that exposure is proportional to free-in-air dose, GE determination should

Table 5 Comparison of GE values derived using the film method with previously published TLD measurement results obtained using the same MDCT scanner. Tabulated values correspond to body mode of operation.

\begin{tabular}{|c|c|c|c|c|c|c|c|c|c|c|}
\hline \multirow[t]{3}{*}{ Collimation (mm) } & \multicolumn{4}{|c|}{$80 \mathrm{kVp}$} & \multicolumn{6}{|c|}{$120 \mathrm{kVp}$} \\
\hline & \multicolumn{2}{|l|}{ SF } & \multicolumn{2}{|l|}{ LF } & \multicolumn{3}{|l|}{ SF } & \multicolumn{3}{|l|}{ LF } \\
\hline & Film & TLDs $^{a}$ & Film & TLDs $^{a}$ & Film & TLDs $^{a}$ & $\operatorname{ImpaCT}^{\mathrm{b}}$ & Film & TLDs $^{\mathrm{a}}$ & ImpaCT $^{\mathrm{b}}$ \\
\hline 1.2 & 50.9 & 42.2 & N/A & 29.5 & 52.2 & 47.0 & 54 & N/A & 30.8 & N/A \\
\hline 2 & 70.0 & 53.1 & 55.9 & 38.2 & 70.2 & 57.1 & 72 & 57.3 & 41.9 & N/A \\
\hline 9 & 74.4 & 69.8 & 69.4 & 64.2 & 73.0 & 74.0 & 76 & 67.6 & 67.6 & 69 \\
\hline 10 & 89.2 & 82.2 & 86.8 & 82.8 & 90.6 & 88.1 & 93 & 87.1 & 87.3 & 92 \\
\hline 12 & 77.5 & 70.8 & 72.6 & 68.6 & 77.4 & 76.9 & 83 & 72.7 & 73.1 & 83 \\
\hline 18 & 83.2 & 82.1 & 81.0 & 79.4 & 84.8 & 84.8 & 86 & 81.3 & 80.3 & 86 \\
\hline 24 & 86.2 & 85.1 & 83.3 & 81.8 & 85.7 & 87.1 & 91 & 82.6 & 81.9 & 86 \\
\hline
\end{tabular}

SF: Small focus; LF: Large focus; TLDs: Thermoluminescent dosimeters; N/A: Not available.

${ }^{a}$ Reference [9].

b Reference [10]. 


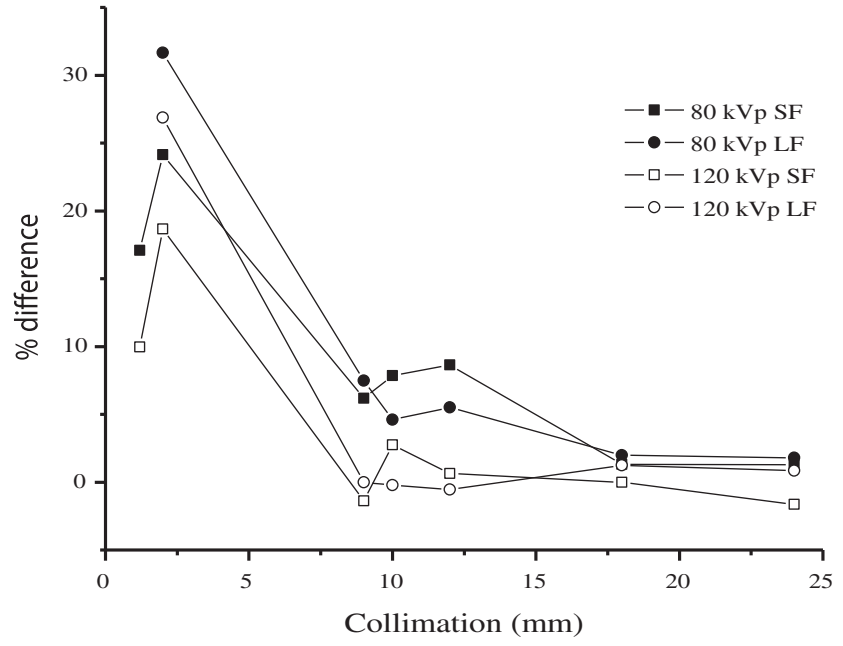

Figure 9 Percentage difference of GE values measured with TLDs from corresponding values obtained with the film method. Measurements were performed for 80 and $120 \mathrm{kVp}$, body mode of operation, for the large (LF) and the small focal spot (SF).

be possible. Equations of pixel value versus exposure for various CR systems are available in the literature [19]. Once radiation profiles were obtained the procedure followed to finally calculate GE would be similar to that followed in film measurements. Additionally, in contrast to radiographic films, CR image plates are reusable.

Variance observed among the values obtained with the various methods could be attributed to the inherent uncertainties of each method. A source of error for film measurements was the film processing system. Films developed at various occasions had different fog, depending on developer temperature and chemical composition which cannot be easily reproduced [20]. Thus care should be taken to subtract the proper background dose from the digitized film images, corresponding to the Base + fog OD at the time of film development. Base + fog OD can be determined by developing an unexposed film every time a parameter that affects fog changes. Failing to subtract the proper background could result to a fluctuation in the values of GE. This fluctuation is expected to be larger for thin beams because the penumbral region is a larger fraction of the beam width compared to wide beams. The CT-

Table 6 Qualitative advantages and disadvantages of the methods to determine GE discussed in the present study.

\begin{tabular}{llll}
\hline $\begin{array}{l}\text { Measurement } \\
\text { method }\end{array}$ & $\begin{array}{l}\text { Dose profile } \\
\text { spatial sampling } \\
\text { (thin beams) }\end{array}$ & $\begin{array}{l}\text { Dose profile } \\
\text { spatial sampling } \\
\text { (wide beams) }\end{array}$ & $\begin{array}{l}\text { Ease } \\
\text { of use }\end{array}$ \\
\hline Film & + & + & - \\
CT-SD16 & + & + & + \\
TLDs & - & + & - \\
CR & + & + & + \\
\hline
\end{tabular}

TLDs: Thermoluminescent dosimeters; CR: Computed radiography.
SD16 solid state detector measures dose profiles in an automated way with no margin for systematic errors attributable to the user. Thus variance observed over repeated measurements should be considered inherent to the measurement system. Similar to film, TLD measurements are also realized in many steps. If all procedures are carried out with the required caution, repeatability should be acceptable. Since no TLD measurements were carried out in the course of this study the repeatability of TLD measurements was not assessed.

It is worth mentioning that GE values obtained with film were generally higher than the corresponding values measured with the CT-SD16 detector. This discrepancy may be attributed to the finite thickness of the solid state detector (i.e. $0.25 \mathrm{~mm}$ ) which causes a blurring at the edges of the dose profiles resulting in a slight decrease of the estimated GE. This effect is even worse when stacked TLD chips are used due to higher detector thickness (i.e. $0.9 \mathrm{~mm}$ ). The decrease in the estimated GE due to limited spatial sampling is, as expected, more prominent for thin beams.

Summarizing, Table 6 gives an overview of the pros and cons of all methods discussed in the present study. The parameters considered are the dose profile spatial sampling and the ease of use of each method. A "+" or "-" means that the parameter in question is an advantage or a disadvantage of the method respectively. In case radiographic film or TLDs are not available, the use of self-developing radiochromic films or CR could be used alternatively for GE measurements. Indeed, published experience suggests that determination of GE using self-developing radiochromic film or CR equipment may be also feasible $[21,22]$. However, further studies are needed to systematically investigate the efficacy of these methods.

\section{Conclusions}

The radiographic film method and the method based on a new solid state detector dedicated for CT dosimetry are capable of collecting detailed dose profiles and may be used to efficiently assess the GE of either thin or wide beam collimations used in modern MDCT scanners. The solid state detector is very easy to use compared to film but its inability to measure dose profiles of beams not available in spiral mode constitutes an important drawback. The TLD method is by far the most tedious. Furthermore, TLD stacks' spatial sampling is comparatively low and cannot accurately represent the shape of the dose profile for narrow beam collimations in the same detail achieved by films or the CT-SD16.

\section{Conflict of interest statement}

None of the authors of the present manuscript has declared any conflict of interest.

\section{References}

[1] Lewis MA. Multislice CT: opportunities and challenges. Br J Radiol 2001;74:779-81. 
[2] Toth TL. Dose reduction opportunities for CT scanners. Pediatr Radiol 2002;32:261-7.

[3] Kalra MK, Maher MM, Toth TL, Hamberg LM, Blake MA, Shepard JA, et al. Strategies for CT radiation dose optimization. Radiology 2004;230:619-28.

[4] International Electrotechnical Commission. International Standard Amendment 1 to IEC 60601-2-44, edition 2: medical electrical equipment, part 2-44: particular requirements for the safety of $\mathrm{x}$-ray equipment for computed tomography 1-7. Geneva, Switzerland: IEC; 2002.

[5] Lewis M. Radiation dose issues in multi-slice CT scanning. Imaging Performance Assessment of CT Scanners Group (ImPACT), technology update no. 3. Norwich, UK: Crown Copyright; 2005.

[6] Brenner DJ, Hall EJ. Computed tomography - an increasing source of radiation exposure. N Engl J Med 2007;357(22): 2277-84.

[7] Hansen J, Jurik AG. Analysis of current practice of CT examinations. Acta Oncol 2009;48:295-301.

[8] Smith-Bindman R, Lipson J, Marcus R, Kim KP, Mahesh M, Gould $R$, et al. Radiation dose associated with common computed tomography examinations and the associated lifetime attributable risk of cancer. Arch Intern Med 2009; 169(22):2078-86.

[9] Perisinakis K, Papadakis AE, Damilakis J. The effect of $x$ ray beam quality and geometry on radiation utilization efficiency in multi-detector CT imaging. Med Phys 2009;36: 1258-66.

[10] Platten D, Keat N, Lewis M, Barrett J, Edyvean S. Siemens Sensation $16 \mathrm{CT}$ scanner technical evaluation. Imaging Performance Assessment of CT scanners group (ImPACT). Medicines and Healthcare products Regulatory Agency (MHRA) Evaluation Report, MDA 04037. Norwich, UK: Crown Copyright; 2004.

[11] Goldman LW. Principles of CT: radiation dose and image quality. J Nucl Med Technol 2007;35:213-25.

[12] Liu Ho-Ling, Liu RR, Reeve DM, Sheppard SJ, Willis CE. Measurement of $C T$ radiation profile width using $C R$ imaging plates. Med Phys 2005;32:2881-7.
[13] Theocharopoulos N, Perisinakis K, Damilakis J, Karampekios S, Gourtsoyiannis N. Dosimetric characteristics of a 16-slice computed tomography scanner. Eur Radiol 2006;16:2575-85.

[14] Niroomand-Rad A, Blackwell CR, Coursey BM, Gall KP, Galvin JM, McLaughlin WL, et al. Radiochromic film dosimetry. Report of the American Association of Physicists in Medicine (AAPM), Task group No. 55 (AAPM Report No. 63). College Park, MD: AAPM; 1998.

[15] Kalender W. Dose management in multi-slice spiral computed tomography. Eur Radiol Syllabus 2004;14:40-9.

[16] Toth TL, Bromberg NB, Pan Tin-Su, Rabe J, Woloschek SJ, $\mathrm{Li}$ Jianying, et al. A dose reduction $\mathrm{x}$-ray beam positioning system for high-speed multislice CT scanners. Med Phys 2000;27:2659-68.

[17] Kobayashi M, Koshida K, SuzukiS, Katada K. Evaluation of geometric efficiency and radiation exposure in z-axis for volume scan. Radiat Prot Dosim; 2010, http://dx.doi.org/10.1093/rpd/ncq283. Advance access [accessed 18/01/12].

[18] Dixon RL, Anderson JA, Bakalyar DM, Boedeker K, Boone JM, Cody DD, et al. Comprehensive methodology for the evaluation of radiation dose in $\mathrm{x}$-ray computed tomography. Report of the American Association of Physicists in Medicine (AAPM), Task group No. 111 (AAPM Report No. 111). College Park, MD: AAPM; 2010.

[19] Samei E, Seibert JA, Willis CE, Flynn MJ, Mah E, Junck KL. Performance evaluation of computed radiography systems. Med Phys 2001;28:361-71.

[20] Suleiman OH, Barnes GT, Bunch PC, Butler PF, Gray JE, Haus AG, et al. Validating Automatic film Processor Performance. Report of the American Association of Physicists in Medicine (AAPM), Task group No. 22 (AAPM Report No. 94). College Park, MD: AAPM; 2006.

[21] Gorny KR, Leitzen SL, Bruesewitz MR, Kofler JM, Hangiandreou NJ, McCollough $\mathrm{CH}$. The calibration of experimental self-developing Gafchromic ${ }^{\circledR}$ HXR film for the measurement of radiation dose in computed tomography. Med Phys 2005;32:1010-6.

[22] Rampado O, Garelli E, Ropolo R. Computed tomography dose measurements with radiochromic films and a flatbed scanner. Med Phys 2010;37:189-96. 